\title{
THE RADIAL HEAT EQUATION WITH POLE TYPE DATA ${ }^{1}$
}

BY L. R. BRAGG

Communicated by A. Zygmund, August 22, 1966

1. Introduction. Recently, detailed studies have been undertaken relating to the solutions and expansions of solutions of the initial value problem

$$
\begin{aligned}
& \text { (a) } U_{t}(r, t)=\Delta_{\mu} U(r, t), \quad r>0, t>0, \\
& \text { (b) } U(r, 0)=\phi(r)
\end{aligned}
$$

with $\Delta_{\mu} \equiv D_{r}^{2}+[(\mu-1) / r] D_{r}$. Results have been obtained when $\phi(r)$ is entire of growth $(1, \sigma)$ in $r^{2}$ [1], [3], [4] and these have been extended to the $L_{2}$ theory in [3]. In this note, we state some results on the structures of solutions of (1) when the data function $\phi(r)$ has a pole at $r=0$ but is otherwise entire. These structures are defined in terms of convolution integrals and the proofs are based on the Laplace transform formulation [2] of solutions of (1) and the expansion theory referred to above. The details of the proofs will appear in a forthcoming paper that will also discuss logarithmic singularities.

We denote by $U^{\mu}(r, t ; \phi(r))$ the solution of (1) defined by

$$
\int_{0}^{\infty} K_{\mu}(r, \xi ; t) \phi(\xi) d \xi
$$

with

$$
K_{\mu}(r, \xi ; t)=\frac{1}{2 t} r^{1-\mu / 2 \xi \xi^{\mu / 2}} \exp \left[-\left(r^{2}+\xi^{2}\right) / 4 t\right] I_{\mu / 2-1}(r \xi / 2 t) .
$$

(See [1], [4].) The abbreviation $a=r^{2} / 16 t^{2}$ will be used in the statement of results.

2. Main results. Our first theorem relates to functions $\phi(r)$ that are odd while the remaining results relate strictly to functions with poles.

THEOREM 1. Let $\phi(r)=r \psi(r)$ in which $\psi(r)$ is an entire function of $r^{2}$ of growth $(1, \sigma)$. For $0 \leqq t<1 / 4 \sigma$ and $\mu>2$,

1 This research was supported by the National Aeronautics and Space Administration Grant No. NsG-544. 
$U^{\mu}(r, t ; \phi(r))$

$$
=\frac{r^{2-\mu} \exp \left[-r^{2} / 4 t\right]}{\pi^{1 / 2}(4 t)^{5 / 2-\mu}} \int_{0}^{a}(a-\xi)^{-1 / 2} \xi^{(\mu-8) / 2} e^{4 \xi t} U^{\mu-1}\left(4 t \xi^{1 / 2}, t ; \psi\right) d \xi .
$$

THEOREM 2. Let $\phi(r)=r^{2-\mu-2 \alpha} \psi(r)$ with $0 \leqq \alpha<1 / 2$ and $\psi(r)$ an entire function of $r^{2}$ of growth $(1, \sigma)$. For $0 \leqq t<1 / 4 \sigma$ and $\mu>2$

$$
\begin{aligned}
U^{\mu}(r, t ; \phi(r))= & \frac{r^{2-\mu} \exp \left[-r^{2} / 4 t\right](4 t)^{\mu / 2-\alpha-1}}{\Gamma(\mu / 2+\alpha-1)} \\
& \cdot \int_{0}^{a} \xi^{-\alpha}(a-\xi)^{\mu / 2+\alpha-2} e^{4 \xi t} U^{2-2 \alpha}\left(4 t \xi^{1 / 2}, t ; \psi\right) d \xi .
\end{aligned}
$$

Observe that the choice $\alpha=0$ in Theorem 2 corresponds to the case in which the multiplier of $\psi(r)$ is precisely the potential function for the Laplacian operator $\Delta_{\mu}$. This theorem shows that the pole can be more badly behaved than the potential function. In fact, the following theorem shows that the pole can be as badly behaved as $r^{-\mu+\epsilon}$ for arbitrary $\epsilon>0$ and still give rise to a classical solution.

TheOREM 3. Let $\phi(r)=r^{2-\mu-2 \alpha}\left\{A+r^{2} \psi(r)\right\}$ in which $\alpha$ is close to but less than $1, \mu / 2+\alpha>2, A$ is a constant, and $\psi(r)$ is an entire function of $r^{2}$ of growth $(1, \sigma)$. For $0 \leqq t<1 / 4 \sigma$,

$$
\begin{aligned}
& U^{\mu}(r, t ; \phi)=\frac{r^{2-\mu} \exp \left[-r^{2} / 4 t\right]}{(4 t)^{1-\mu / 2}} \\
& \cdot\left\{\frac{A(4 t)^{1-\alpha}}{\Gamma(\mu / 2+\alpha-1)} \int_{0}^{a} \xi^{-\alpha}(a-\xi)^{\mu / 2+\alpha-2} e^{4 \xi t} d \xi+\frac{(4 t)^{2-\alpha}}{\Gamma(\mu / 2+\alpha-2)}\right. \\
& \left.\cdot \int_{0}^{a} \xi^{1-\alpha}(a-\xi)^{\mu / 2+\alpha-3} e^{4 \xi t} U^{4-2 \alpha}(4 t \sqrt{\xi}, t ; \psi) d \xi\right\} .
\end{aligned}
$$

It follows, from the change of valuables $\xi=a \sigma$, that

$$
\lim _{r \rightarrow 0 ; t>0} U^{\mu}(r, t ; \phi)
$$

exists in all of the above theorems. This simply states that the pole in the data function dissipates from the solution function.

Finally, as a corollary to Theorem 2 where $\alpha=0$, we have the special result:

CoRollary 2.1. Let $\mu=2 m$ be an even integer with $m \geqq 2$. Then 


$$
U^{2 m}\left(r, t ; r^{2-2 m+2 j}\right)=r^{2-2 m} R_{j}^{4-2 m}(r, t)+(-1)^{j+1} \exp \left[-r^{2} / 4 t\right]
$$

$$
\cdot \sum_{k=0}^{m-2-j} \frac{(m-2-k) !}{k !(m-2-j-k) !} r^{2(1-m+k)}(4 t)^{j-k}, 0 \leqq j \leqq m-2 .
$$

In this, $R_{\mu}^{4-\mu}(r, t)=j !(4 t)^{j} L_{j}^{(1-\mu / 2)}\left(-r^{2} / 4 t\right)$ with $L_{j}^{\nu}(x)$ the generalized Laguerre polynomial of degree $j$ and index $\nu$. In the case that $\mu$ is even with $\mu \geqq 4$, we can divide the data into the pole type terms (finite in number) and the entire part. The corollary applies to the pole terms while the expansion theory in [1], [4] applies to the entire part.

\section{REFERENCES}

1. L. R. Bragg, The radial heat polynomials and related functions, Trans. Amer. Math. Soc. 119 (1965), 270-290.

2. - The radial heat equation and Laplace transforms, SIAM J. Appl. Math. (to appear).

3. D. T. Haimo, Functions with the Huygen's property, Bull. Amer. Math. Soc. 71 (1965), 528-532.

4. - Expansions in terms of generalized heat polynomials and of their Appell transforms, J. Math. Mech. 15 (1966), 735-758.

Case Institute of Technology and

OAKLAND UNIVERSITY 